Canadian Oncology

Nursing Journal

Revue canadienne

de soins infirmiers

en oncologie

Volume 28, Issue 2 • Spring 2018

elSSN: 2368-8076 


\section{Autonomisation des survivants du cancer pour répondre à leurs besoins physiques et psychosociaux : évaluation d'un projet}

par Carrie M. Liska, Robin Morash, Lise Paquet et Dawn Stacey

\section{RÉSUMÉ}

Notre Programme de bien-être au-delà du cancer a été établi et évalué en tant que projet d'amélioration de la qualité. Les plans de soins individualisés pour les survivants et les fournisseurs de soins primaires incluaient des recommandations sur la surveillance $d u$ cancer et sur les besoins physiques et psychosociaux signalés par les survivants. Au rendez-vous de transition, une infirmière en oncologie a passé en revue le plan de soins et les stratégies de gestion des symptômes avec les survivants. Nous avons évalué les besoins physiques et psychosociaux, et le sentiment d'autonomie de 70 survivantes du cancer du sein et de 53 survivants du cancer colorectal à l'adhésion au programme, puis une année après la transition aux soins primaires. Les survivants ne recevaient plus de traitement actif depuis des mois, voire des années (10 ans et plus), mais les survivants $d u$ cancer colorectal ont été aiguillés plus tôt (1,2 an en moyenne). Au départ, on a observé que les survivants du cancer colorectal s'inquiétaient peu de leurs besoins (scores < 1,0 sur 5,0) et que les survivantes du cancer du sein avaient quant à elles quelques préoccupations liées aux troubles du sommeil, aux changements pondéraux, aux effets sur la mémoire/concentration et à la peur de la récidive (scores de 1,0 à 1,5 sur 5,0). Tous les survivants ont rapporté s'être sentis bien autonomisés (> 3,0 sur 4,0). Les besoins et les niveaux d'autonomisation étaient pour la plupart les mêmes après une année. On observe statistiquement que les survivants du cancer colorectal avaient davantage peur de la récidive après un an. En

\section{AU SUJET DES AUTEURES}

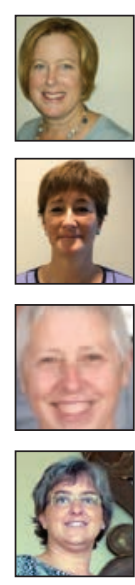

Carrie M. Liska inf. aut. M.Sc.inf., L'Hôpital d'Ottawa, Ottawa, ON

Robin Morash, inf. aut. M.Sc.S., L'Hôpital d'Ottawa, Ottawa, ON

Lise Paquet, Ph.D., Université Carleton, Ottawa, ON

Dawn Stacey, inf. aut. Ph.D., Université d’Ottawa, Ottawa, ON

Auteure-ressource : Carrie M. Liska, inf. aut. M.Sc.inf., Coordonnatrice des soins, Programme de cancérologie de l'Hôpital d'Ottawa, 501, Smyth Road, Ottawa (Ont.) K1H 8 L6

Tél. : 613-737-7700, poste 70264; Téléc. : 613-739-6965;

Courriel : cliska@toh.on.ca

DOI:10.5737/236880762828288 résumé, les survivants du cancer dotés d'un plan de soins continuent de se sentir autonomisés un an après avoir reçu leur congé.

Mots clés : cancer, survie au cancer, survivants, autonomisation, fournisseurs de soins primaires

\section{CONTEXTE}

T e cancer est maintenant envisagé comme une maladie chroLnique exigeant une offre de soins post-traitement à long terme de qualité élevée (Drayton et al., 2012; Phillips et Currow, 2010). En 2016, 202400 Canadiens ont reçu un diagnostic de cancer et, grâce aux améliorations en dépistage précoce et traitement, on s'attend que $60 \%$ d'entre eux survivent à la maladie pendant au moins cinq ans (Statistiques canadiennes sur le cancer, 2016). Par conséquent, les soins de survie à long terme s'imposent progressivement comme un fardeau dans les soins de santé et impliquent de nombreuses ressources comme des soins psychosociaux et des soins de soutien, la promotion de la santé, la surveillance des séquelles tardives et des séquelles à long terme, la surveillance par un suivi et le dépistage d'autres cancers (Statistiques canadiennes sur le cancer, 2016; Howell et al., 2011; McCorkle, 2011; Morgan, 2009). La tendance à recourir à des modèles communautaires de soins aux survivants dirigés par des fournisseurs soins primaires s'est donc accrue (Dawes et al, 2015). Malheureusement, on en sait peu sur les besoins psychosociaux des patients après leur transition aux soins primaires (Hebdon et al., 2014; Lewis et al., 2009).

Un effet psychosocial important des soins de survie au cancer est l'autonomisation du patient (Jerofke, 2013; McCorkle, 2011; Kuijpers, 2013; Lauzier et al., 2014; van den Berg et al., 2013). Lautonomisation est ressentie comme une attitude que certains patients possèdent lorsqu'ils sont davantage impliqués dans le traitement de leur maladie; un patient autonomisé se montre proactif dans la recherche de solutions et pose des questions pour gérer sa maladie (Bulsara, Stiles, Ward et Bulsara, 2006; Bulsara et Styles, 2013). Autonomiser les patients ayant une maladie chronique est crucial pour les aider à aborder la maladie, à y faire face et à réduire la détresse psychologique (Bulsara, Stiles, Ward et Bulsara, 2006). Suivant le Modèle sur le soin des maladies chroniques (Wagner, 2001), un soutien à l'autogestion aide à autonomiser et à préparer les patients à gérer leurs soins de santé. Les stratégies d'autogestion proposées par les prestataires de soins de santé incluent des interventions d'éducation et de soutien pour accroître les habiletés et la confiance du patient pour gérer ses problèmes de santé (Adams, Greiner et Corrigan, 2004; McCorkle, 2011).

Il y a malheureusement peu de recherches sur l'autonomisation à l'étape des soins de survie au cancer, post-traitement, et ces 
dernières reposent sur des mesures indirectes, soit la façon dont les infirmières perçoivent l'autonomie acquise (Mok, Martinson et Wong, 2004; van Uden-Kraan et al., 2008) ou l'évaluation de concepts connexes comme la qualité de vie, l'anxiété, la gestion des symptômes, l'acquisition de connaissances et les habiletés d'adaptation (Ryhänen et al., 2013; Kane, Boland et Herst, 2014; Pereira, 2014; Jerofke, 2013; Knobf, 2013; McCarley, 2009; Morgan, 2009), plutôt que de mesurer plus directement le point de vue des premiers intéressés, les survivants (McAllister et al., 2012). Par conséquent, on ne sait pas si les programmes encadrant la transition des survivants au cancer à des soins primaires de suivi autonomisent adéquatement les patients pour qu'ils se montrent proactifs dans leurs questions et dans la recherche de solutions pour gérer leurs besoins durant la phase de survie de leur expérience globale du cancer (Bulsara et Styles, 2013).

En 2006, le rapport From Cancer Patient to Cancer Survivor: Lost in Transition de l'Institute of Medicine contenait quatre recommandations sur les soins de survie : a) avoir des stratégies pour prévenir les séquelles tardives, y compris la récidive; b) offrir une surveillance de l'apparition de métastases; c) proposer des interventions sur les conséquences du cancer et son traitement; d) coordonner les soins entre les spécialistes et les prestataires de soins primaires.

On a demandé aux centres de cancérologie d'intégrer ces quatre éléments clés aux soins de survie. Les normes d'Agrément Canada exigent aussi que les prestataires de soins de santé communiquent efficacement entre eux au moment de transférer les soins aux patients pour prévenir tous malentendus; on s'attend notamment à ce que les patients et les familles reçoivent de l'information standardisée de haute qualité et basée sur des données probantes pour prendre des décisions et prendre soin d'eux (Agrément Canada et Organisation de normes en santé, 2017). Reconnaissant le besoin d'intégrer les soins de survie pour répondre aux besoins uniques des survivants tout en tenant compte des recommandations de l'Institute of Medecine (2006) et des lignes directrices en matière de surveillance d'Action Cancer Ontario (2012), le centre de cancérologie de l'Hôpital d'Ottawa a conçu son Programme de bien-être au-delà du cancer pour fournir aux patients des soins de survie de grande qualité à la fin de leur traitement actif. Ce programme a été lancé en mars 2012 pour les survivants du cancer colorectal et de l'endomètre, et en février 2013 pour les survivantes du cancer du sein.

\section{BUT DE L'ÉTUDE}

Lobjectif de ce projet était de décrire les besoins physiques et psychosociaux des survivants au cancer colorectal et des survivantes au cancer du sein, de même que leur sentiment d'autonomie au début du Programme de bien-être au-delà du cancer et un an après avoir été transférés aux soins primaires. Des objectifs spécifiques ont été établis pour déceler : a) les changements dans les besoins physiques et psychosociaux signalés par les survivants un an après avoir été transférés aux soins primaires; b) les changements dans le sentiment d'autonomie un an après avoir été transférés aux soins primaires; c) pour tout changement observé, si les changements dans le sentiment d'autonomie étaient liés aux changements de besoins physiques et psychosociaux.

\section{MÉTHODOLOGIE}

Un projet d'amélioration de la qualité a été mené auprès des patients recommandés au programme entre mars 2012 et juin 2013. Ce genre de projet découle souvent de discussions entre cliniciens et vise à évaluer les pratiques cliniques pour déterminer s'il faut modifier la pratique (Polit et Beck, 2012). Ce projet d'amélioration de la qualité a été passé en revue par le président du Conseil d'éthique de la recherche du Réseau des sciences de la santé d'Ottawa (CER) qui a reconnu qu'il s'agissait d'un projet d'amélioration de la qualité ne requérant pas l'approbation du CER.

\section{Cadre de l'étude}

Le Programme de bien-être au-delà du cancer est un programme ambulatoire qui s'adresse aux survivants du cancer d'un grand hôpital universitaire servant une population de 1,3 million de personnes dans l'Est ontarien. Les patients transférés aux soins primaires sont recommandés par des oncologues à la fin de leur traitement actif, lorsque les symptômes sont stables et ne posent aucune inquiétude du point de vue médical, et que le risque de récidive est jugé faible. Le Programme de bien-être au-delà du cancer a été conçu en se basant sur le Cadre des soins de soutien (Fitch, 2008) et les principes du Modèle sur le soin des maladies chroniques (Wagner, 2001). L'objectif du programme est d'assurer aux patients l'accès à des ressources et à du soutien à la fin de leur traitement actif pour : a) répondre à leurs besoins physiques et psychosociaux subsistants; b) susciter leur désir de participer, avec leur prestataire de soins primaires, à la gestion de leurs soins de suivi.

\section{Participants à l'étude}

Lorsque le programme a été lancé, beaucoup de survivants du cancer qui étaient suivis depuis de nombreuses années au centre de cancérologie ont été invités à adhérer au Programme de bien-être au-delà du cancer pour que le suivi des soins de surveillance soit transféré à leur prestataire de soins primaires. Les patients admissibles avaient un cancer colorectal ou un cancer du sein; ils avaient terminé leur traitement actif contre le cancer et le risque de récidive était faible.

\section{PROCÉDURES}

On demande régulièrement aux patients recommandés au Programme de bien-être au-delà du cancer de remplir une évaluation des besoins physiques et psychosociaux, ainsi que le questionnaire de Bulsara et collègues (2006) comportant une échelle sur l'autonomisation des patients. Les patients se soumettent alors quatre interventions clés pendant qu'ils participent au Programme de bien-être au-delà du cancer.

Premièrement, les patients sont invités à suivre une formation didactique en classe d'une durée de deux heures sur des données portant spécifiquement sur la maladie. Les sujets abordés sont la coordination des soins au moment de la transition, le dépistage d'autres cancers, les saines habitudes de vie, la gestion des symptômes des plus fréquentes séquelles physiques et psychosociales tardives et à long terme après un traitement contre le cancer, et le processus d'une réintégration ultérieure au programme en oncologie si nécessaire. 
Deuxièmement, durant la formation éducative, les patients reçoivent de la documentation écrite portant sur leur maladie et un accès à des ressources disponibles en ligne sur les séquelles tardives et les séquelles à long terme.

Troisièmement, un plan de soins de survie individualisé est préparé par une infirmière en oncologie qui passe en revue l'équipe de soins oncologiques du patient, résume la maladie et le traitement, et donne les lignes directrices de la surveillance durant le suivi. Les besoins physiques et psychosociaux signalés par les patients, qui sont indiqués comme allant de modérés à extrêmes dans lévaluation des besoins, sont inclus dans le plan de soins.

Quatrièmement, tous les patients sont invités à se présenter à un rendez-vous de transition de 30 minutes pour passer en revue le plan de soins avec une infirmière en oncologie. Un exemplaire du plan de soins est passé en revue et remis au patient, et l'infirmière discute des stratégies de gestion des symptômes pour répondre aux besoins physiques et psychosociaux subsistants soulignés. Le verbatim du rendez-vous de transition, une lettre de transition et un exemplaire du plan de soins sont envoyés au prestataire de soins primaires des patients. Le numéro de téléphone du Programme de bienêtre au-delà du cancer est aussi fourni au prestataire des soins primaires dans l'éventualité où des questions ou inquiétudes seraient soulevées et pour assurer si nécessaire une réintégration rapide dans le Programme de bien-être au-delà du cancer.

On notera qu'uniquement aux fins du projet d'amélioration de la qualité, l'évaluation des besoins et le questionnaire sur l'autonomisation des patients en fonction d'une échelle ont été postés aux patients une année après la date de recommandation au Programme de bien-être au-delà du cancer, avec invitation à répondre au questionnaire et à le retourner.

\section{Outils de collecte de données}

Les outils de collecte de données ont été choisis en suivant le Cadre des soins de soutien (Fitch, 2008) et le Modèle sur le soin des maladies chroniques (Wagner, 2001). Le questionnaire sur l'évaluation des soins est une adaptation de l'Évaluation des besoins des survivants $d u$ cancer réalisée grâce à une permission spéciale du Programme d'enseignement sur le cancer de la clinique Mayo (Mayo Clinic Cancer Center, s.d.). Ce questionnaire compte 50 items utilisés pour évaluer les symptômes physiques et psychosociaux sur une échelle de Likert en six points, où 0 indique « aucune inquiétude » et 5 une « inquiétude extrême ». Cet outil a été utilisé pour cerner les besoins en soins de soutien physiques et psychosociaux. Aux fins de la présente étude, nous nous sommes concentrés seulement sur les neuf besoins physiques et psychosociaux clés signalés par les patients du Programme de bien-être au-delà du cancer et reconnus comme étant les plus problématiques pour les survivants du cancer du sein et du cancer colorectal (Rushton et al., 2015; Schlairet, Heddon et Griffis, 2010).

L'échelle de Bulsara sur l'autonomisation des patients atteints d'un cancer compte 15 énoncés évalués sur une échelle de Likert en quatre points allant de «Tout à fait en désaccord » à « Tout à fait d'accord » (Bulsara et al., 2006). Un score plus élevé indique un sentiment d'autonomie également élevé. Le coefficient alpha de Cronbach pour l'échelle sur l'autonomisation était 0,85 au départ et de 0,90 au suivi, un très bon coefficient de cohérence interne. L'analyse des principaux éléments indiquait que les items sélectionnés avaient des factorielles similaires $>, 66$.

\section{Analyse}

Toutes les données de l'évaluation des besoins physiques et psychosociaux et de l'échelle sur l'autonomisation ont été entrées dans le logiciel SPSS (Statistical Package for the Social Sciences). Les résultats pour les survivants du cancer colorectal et du cancer du sein ont été analysés séparément, à partir des mêmes méthodes. Dans un premier temps, des t-tests dépendants ont servi à mesurer les changements en matière de besoins et d'autonomisation au fil du temps. Si un suivi s'avérait nécessaire à la fin des premières analyses, des analyses de corrélation étaient alors menées pour examiner si les changements pour l'autonomisation étaient corrélés à des changements en matière de besoins.

\section{RÉSULTATS}

La fourchette d'âge et l'état civil des survivants du cancer du sein et du cancer colorectal étaient similaires. Chez les survivants du cancer colorectal, 1,2 année en moyenne s'était écoulée depuis la fin du traitement actif (entre 1 mois et 10 ans). Chez les survivantes du cancer du sein, 4,6 années (entre 2 ans et 17 ans) s'étaient écoulées depuis leur dernier traitement actif (voir Tableau 1).

\begin{tabular}{|l|c|c|}
\hline \multicolumn{3}{|l|}{ Tableau 1: Caractéristiques des patients } \\
\hline Sujet & Colorectal N=53 & Sein N=70 \\
\hline Tranche d'âge (échelle) & $38-90$ & $42-92$ \\
\hline$<49$ années & 2 & 7 \\
\hline $50-59$ & 8 & 18 \\
\hline $60-69$ & 12 & 19 \\
\hline $70-79$ & 20 & 17 \\
\hline$>80$ & 11 & 9 \\
\hline Genre : & & \\
\hline Homme & 35 & 0 \\
\hline Femme & 18 & 70 \\
\hline État civil : & 37 & 43 \\
\hline Marié/en couple & 16 & 17 \\
\hline Célibat/veuvage & 1 mois à 10 ans & 2 à 17 ans \\
\hline Durée post-traitement (échelle) & 35 & 13 \\
\hline$<1$ an & 8 & 7 \\
\hline $1-2$ ans & 5 & 7 \\
\hline$>2-3$ ans & 3 & 13 \\
\hline$>3-5$ ans & 1 & 25 \\
\hline$>5-10$ ans & 1 & 5 \\
\hline$>10$ ans & & \\
\hline
\end{tabular}




\begin{tabular}{|c|c|c|c|}
\hline $\begin{array}{l}\text { Tableau } 2 \text { : Inquiétude des st } \\
\text { psychosociaux }\end{array}$ & ants sur le & besoins ph & ques et \\
\hline A : Groupe du cancer colore & $(N=53)$ & & \\
\hline Domaine & $\begin{array}{l}\text { Seuil de } \\
\text { référence }\end{array}$ & 1 an après & valeur $p$ \\
\hline & $\begin{array}{l}\text { Moyenne } \\
\text { (éc.-t.) }\end{array}$ & $\begin{array}{l}\text { Moyenne } \\
\text { (éc.-t.) }\end{array}$ & \\
\hline Picotements (mains/pieds) & $0,79(1,32)$ & $0,88(1,35)$ & 0,647 \\
\hline Problèmes sexuels & $0,87(1,29)$ & $0,83(1,31)$ & 0,796 \\
\hline Troubles du sommeil & $0,88(1,26)$ & $0,74(1,03)$ & 0,398 \\
\hline Problèmes (intestin/vessie) & $0,94(1,51)$ & $1,04(1,38)$ & 0,601 \\
\hline Fatigue & $0,86(1,02)$ & $1,00(1,06)$ & 0,332 \\
\hline Changements pondéraux & $0,86(1,34)$ & $0,65(1,02)$ & 0,201 \\
\hline Changements corporels & $0,79(1,18)$ & $0,83(1,11)$ & 0,761 \\
\hline Mémoire et concentration & $0,73(1,22)$ & $0,76(1,64)$ & 0,883 \\
\hline Peur de la récidive & $0,79(1,24)$ & $1,31(1,20)$ & 0,005 \\
\hline B : Groupe du cancer du seir & $\mathrm{V}=70)$ & & \\
\hline Domaine & $\begin{array}{l}\text { Seuil de } \\
\text { référence }\end{array}$ & 1 an après & valeur $p$ \\
\hline & $\begin{array}{l}\text { Moyenne } \\
\text { (éc.-t.) }\end{array}$ & $\begin{array}{l}\text { Moyenne } \\
\text { (éc.-t.) }\end{array}$ & \\
\hline Picotements (mains/pieds) & $0,71(1,17)$ & $0,92(1,37)$ & 0,262 \\
\hline Problèmes sexuels & $0,78(1,34)$ & $0,97(1,44)$ & 0,077 \\
\hline Troubles du sommeil & $1,00(1,19)$ & $1,23(1,42)$ & 0,148 \\
\hline Problèmes (intestin/vessie) & $0,68(0,97)$ & $0,83(1,11)$ & 0,335 \\
\hline Fatigue & $0,98(1,20)$ & $1,16(1,36)$ & 0,181 \\
\hline Changements pondéraux & $1,10(1,38)$ & $0,93(1,31)$ & 0,531 \\
\hline Changements corporels & $0,85(1,19)$ & $1,06(1,29)$ & 0,180 \\
\hline Mémoire et concentration & $1,08(1,16)$ & $1,16(1,36)$ & 0,608 \\
\hline Peur de la récidive & $1,46(1,48)$ & $1,50(1,41)$ & 0,775 \\
\hline $\begin{array}{l}\text { Échelle de Likert en six points } \\
5=\text { inquiétude extrême }\end{array}$ & $t$ de $0=a u$ & inquiétude à & \\
\hline
\end{tabular}

\section{Résultats des survivants du cancer colorectal}

Entre mars et septembre 2012, des 131 survivants du cancer colorectal recommandés au Programme de bien-être au-delà du cancer, 126 ont rempli l'évaluation de départ (taux de réponse de $96 \%$ ). Un an plus tard, 125 survivants du cancer colorectal ont reçu le questionnaire et 53 l'ont rempli (taux de réponse de $42 \%$ ). Au départ, les survivants du cancer colorectal avaient indiqué avoir peu d'inquiétude quant à leurs besoins (scores $<1,0$ sur 5,0 ) et indiqué se sentir bien autonomisés (3,00 à 3,76 sur 4,0) (voir Tableaux 2 et 3).

$\mathrm{Au}$ fil du temps, on ne note dans l'ensemble aucun changement statistique significatif dans huit des neuf besoins physiques et psychosociaux chez les survivants du cancer colorectal. Leur peur de la récidive était toutefois passée de

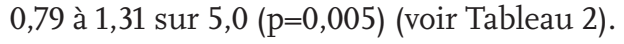

On n'observe au fil du temps aucun changement statistique significatif de l'autonomisation chez les survivants du cancer colorectal (voir Tableau 3). Par conséquent, aucune analyse de corrélation n’a été menée.

\section{Résultats des survivantes du cancer du sein}

Entre janvier et juin 2013, des 164 survivantes du cancer du sein recommandées au Programme de bien-être au-delà du cancer, 163 ont rempli l'évaluation de départ (taux de réponse de $99 \%$ ). Un an plus tard, 163 survivantes du cancer du sein ont reçu le questionnaire et 70 l'ont rempli (taux de réponse de $43 \%$ ). Au départ, les survivantes du cancer du sein avaient indiqué ressentir une certaine inquiétude liée aux troubles du sommeil, aux changements pondéraux, aux mémoire/concentration et à la peur de la récidive (scores de 1,00 à 1,46 sur 5,0) et avaient indiqué se sentir bien autonomisées (3,06 à 3,75 sur 4,0) (Tableaux 2 et 3 ).

On n'observe au fil du temps aucun changement statistique significatif dans les besoins des survivantes du cancer du sein ni dans l'autonomisation. Par conséquent, aucune analyse de corrélation n’a été menée.

\section{DISCUSSION}

Ce projet d'amélioration de la qualité a fait état de niveaux de besoins physiques et psychosociaux faibles chez les survivants du cancer colorectal et du cancer du sein, et d'un sentiment élevé d'autonomie au moment d'adhérer au Programme de bien-être au-delà du cancer. Les besoins physiques et psychosociaux et les niveaux d'autonomisation signalés dans l'ensemble par ces survivants du cancer n'ont montré aucun changement statistique significatif une année après la transition du centre en cancérologie au prestataire de soins primaires. Seuls les survivants du cancer colorectal ont indiqué avoir une plus grande peur de la récidive après un an, parmi les besoins psychosociaux clés. On ne peut clairement dire pourquoi la peur de la récidive s'est accrue dans ce groupe, mais il est intéressant de souligner qu'ils avaient été recommandés au Programme de bienêtre au-delà du cancer plus rapidement après avoir terminé leur traitement actif (1,2 an en moyenne) comparativement au groupe du cancer du sein (4,6 ans en moyenne). Nos 


\begin{tabular}{|c|c|c|c|}
\hline \multicolumn{4}{|l|}{ A : Groupe du cancer colorectal $(\mathrm{N}=53)$} \\
\hline \multirow[t]{2}{*}{ Domaine } & Seuil de référence & 1 an après & \multirow[t]{2}{*}{ valeur $p$} \\
\hline & Moyenne (éc.-t.) & Moyenne (éc.-t.) & \\
\hline Je suis capable de gérer ma maladie. & $3,64(0,486)$ & $3,49(0,547)$ & 0,070 \\
\hline Je dispose de toute l'information requise pour gérer ma maladie. & $3,42(0,753)$ & $3,33(0,674)$ & 0,420 \\
\hline $\begin{array}{l}\text { Je suis capable d'aider les professionnels de la santé à prendre des décisions sur } \\
\text { ma maladie. }\end{array}$ & $3,57(0,547)$ & $3,50(0,552)$ & 0,412 \\
\hline Ma famille m'aide beaucoup. & $3,76(0,480)$ & $3,63(0,610)$ & 0,204 \\
\hline J'ai besoin du soutien de ma famille et de mes amis. & $3,45(0,730)$ & $3,25(0,719)$ & 0,095 \\
\hline Ma famille et mes amis comptent encore sur moi. & $3,30(0,765)$ & $3,07(0,873)$ & 0,086 \\
\hline Je peux m'adapter aux changements dans mes habitudes de vie. & $3,51(0,626)$ & $3,38(0,614)$ & 0,183 \\
\hline $\begin{array}{l}\text { Les professionnels de la santé sont heureux de m'inclure dans les décisions } \\
\text { relatives à ma maladie. }\end{array}$ & $3,66(0,481)$ & $3,50(0,558)$ & 0,136 \\
\hline Je veux que ma famille et mes amis puissent continuer à compter sur moi. & $3,24(0,773)$ & $3,20(0,919)$ & 0,710 \\
\hline Mes amis m'offrent toujours un bon soutien. & $3,50(0,619)$ & $3,40(0,707)$ & 0,341 \\
\hline J'ai le sentiment d'être toujours utile dans ma vie quotidienne. & $3,51(0,585)$ & $3,45(0,583)$ & 0,519 \\
\hline Mes croyances spirituelles m'aident à composer avec la maladie. & $3,29(0,782)$ & $3,37(0,799)$ & 0,519 \\
\hline J'accepte d'avoir à modifier mes habitudes de vie. & $3,20(0,687)$ & $3,23(0,620)$ & 0,850 \\
\hline Des thérapies complémentaires m'aident à composer avec ma maladie. & $3,00(0,791)$ & $3,18(0,809)$ & 0,422 \\
\hline Je fais grandement confiance à mon médecin de famille & $3,60(0,681)$ & $3,70(0,507)$ & 0,280 \\
\hline \multicolumn{4}{|l|}{ B : Groupe du cancer du sein $(N=70)$} \\
\hline \multirow[t]{2}{*}{ Domaine } & Seuil de référence & 1 an après & \multirow[t]{2}{*}{ valeur $p$} \\
\hline & Moyenne (éc.-t.) & Moyenne (éc.-t.) & \\
\hline Je suis capable de gérer ma maladie. & $3,46(0,534)$ & $3,56(0,501)$ & 0,159 \\
\hline Je dispose de toute l'information requise pour gérer ma maladie. & $3,49(0,592)$ & $3,44(0,562)$ & 0,536 \\
\hline $\begin{array}{l}\text { Je suis capable d'aider les professionnels de la santé à prendre des décisions sur } \\
\text { ma maladie. }\end{array}$ & $3,53(0,566)$ & $3,55(0,565)$ & 0,843 \\
\hline Ma famille m'aide beaucoup. & $3,75(0,434)$ & $3,70(0,460)$ & 0,471 \\
\hline J'ai besoin du soutien de ma famille et de mes amis. & $3,50(0,505)$ & $3,55(0,537)$ & 0,518 \\
\hline Ma famille et mes amis comptent encore sur moi. & $3,35(0,641)$ & $3,39(0,559)$ & 0,699 \\
\hline Je peux m'adapter aux changements dans mes habitudes de vie. & $3,54(0,503)$ & $3,46(0,537)$ & 0,301 \\
\hline $\begin{array}{l}\text { Les professionnels de la santé sont heureux de m'inclure dans les décisions } \\
\text { relatives à ma maladie. }\end{array}$ & $3,52(0,504)$ & $3,54(0,539)$ & 0,821 \\
\hline Je veux que ma famille et mes amis puissent continuer à compter sur moi. & $3,35(0,645)$ & $3,38(0,593)$ & 0,687 \\
\hline Mes amis m'offrent toujours un bon soutien. & $3,54(0,502)$ & $3,51(0,536)$ & 0,621 \\
\hline J'ai le sentiment d'être toujours utile dans ma vie quotidienne. & $3,62(0,522)$ & $3,49(0,595)$ & 0,059 \\
\hline Mes croyances spirituelles m'aident à composer avec la maladie. & $3,29(0,733)$ & $3,29(0,731)$ & 1,00 \\
\hline J'accepte d'avoir à modifier mes habitudes de vie. & $3,13(0,672)$ & $3,19(0,704)$ & 0,652 \\
\hline Des thérapies complémentaires m’aident à composer avec ma maladie. & $3,06(0,854)$ & $2,94(0,814)$ & 0,489 \\
\hline Je fais grandement confiance à mon médecin de famille & $3,46(0,727)$ & $3,47(0,796)$ & 0,811 \\
\hline
\end{tabular}

résultats nous amènent à discuter des trois enjeux importants pour améliorer les soins aux survivants du cancer.

Premièrement, définir ce qu'est l'autonomisation du patient dans un contexte de survie au cancer peut éclairer les personnes qui soignent les survivants du cancer en leur donnant une base claire. Une analyse de 67 articles définissant l'autonomisation du patient a révélé des définitions axées sur: a) le processus de transformation à mesure que les patients maîtrisent leur santé et leurs soins de santé pour s'adapter à leur maladie chronique; b) des principes indiquant un état d'autonomie et d'autodétermination; c) les interventions d'autogestion d'une maladie chronique (Bravo, Edwards, Barr, Scholl, Elwyn et McAllister, 2015). Les résultats obtenus chez les patients en fonction de ces définitions étaient l'adaptation à une maladie chronique, la qualité de vie, le bien-être, la satisfaction à l'égard de la vie et l'indépendance. La recherche a 
démontré que les interventions faisant participer les patients et les autonomisant accroissent leur niveau de satisfaction, améliorent leur santé et réduisent les coûts, et on juge essentielle maintenant l'autonomisation des survivants du cancer (Eskildsen et al., 2017).

Deuxièmement, transférer les patients ayant un cancer à faible risque à leurs prestataires de soins primaires s'est déjà avéré sécuritaire et rentable, en plus d'être souvent le choix préféré des patients (Grant, De Rossi et Sussman, 2015). Toutefois, les prestataires de soins primaires rapportent un manque de connaissances, de confiance et de ressources comme des directives sous forme de livret (Dawes et al., 2014; Luctkar-Flude, Aiken, McColl et Tramner, 2015). Ils ont cependant l'occasion d'améliorer leurs connaissances en lisant des directives sur la pratique fondées sur des données probantes, ce qui aide à limiter les tests inutiles et à améliorer la coordination des soins entre les spécialistes en oncologie et les prestataires de soins primaires (Grant, De Rossi et Sussman, 2015; Luctkar-Flude et al., 2015). Dans la présente étude, le plan de soins de survie fourni aux survivants du cancer et à leurs prestataires de soins primaires dans le cadre du Programme de bien-être au-delà du cancer a été conçu pour : offrir un résumé du traitement; donner une orientation fondée sur des données probantes concernant les tests de surveillance de suivi; communiquer les besoins physiques et psychosociaux signalés par les patients; et offrir une orientation sur les stratégies de gestion des symptômes adaptées aux besoins physiques et psychosociaux individuels des survivants du cancer. En même temps que les prestataires de soins primaires étaient accompagnés, le plan de survie au cancer était utilisé pour accroître la compréhension des survivants du cancer quant aux soins de suivi requis et pour les autonomiser en les incitant à participer activement à la gestion de leurs soins (Dawes et al., 2014; Grant, De Rossi et Sussman, 2015; Jabson, 2014; Luctkar-Flude et al., 2015; McCorkle, 2011).

Troisièmement, bien que la littérature soutienne l'autonomisation des survivants par l'éducation, il y a peu de données validant le bénéfice d'interventions spécifiques. Des groupes de soutien en ligne, des interventions en ligne et des cours d'éducation se sont montrés efficaces pour autonomiser des patients atteints du cancer et des patients ayant d'autres maladies chroniques (Kuijpers et al., 2013; Pereira, 2014; van Uden-Kraan et al., 2008). Par conséquent, le Programme de bien-être au-delà du cancer pourrait continuer d'offrir des cours d'éducation et envisager une évaluation plus rigoureuse pour vérifier leur efficacité chez cette population précise de patients, et en particulier chez ceux venant tout juste de terminer leur traitement actif.

\section{LIMITES}

Il faut tenir compte de trois limites principales. Premièrement, la taille des échantillons de survivants du cancer colorectal et du cancer du sein était trop petite pour tirer des conclusions à toute épreuve. Deuxièmement, il y avait parmi les participants une variance dans l'écart temporel entre la fin du traitement actif et la recommandation au Programme de bien-être au-delà du cancer. Cette grande variance pourrait avoir un impact sur les besoins physiques et psychosociaux subsistants signalés par les patients et pourrait influencer leur sentiment d'autonomie. Troisièmement, il faudrait faire des recherches sur chacune des quatre interventions clés utilisées par le Programme de bien-être au-delà du cancer pour déterminer leur efficacité particulière.

\section{CONCLUSION}

La survie au cancer en tant que maladie chronique est une nouvelle perspective et peu d'interventions d'autonomisation ont encore été conçues ou utilisées. Le Programme de bien-être au-delà du cancer a été mis sur pied pour préparer les survivants du cancer à effectuer une transition entre leur oncologue responsable des soins de suivi et leur prestataire de soins primaires. Les quatre interventions clés régulièrement utilisées dans ce programme incluent un cours d'éducation, du matériel éducatif imprimé pour les patients, un plan de soins de survie individualisé en fonction des besoins physiques et psychosociaux uniques des patients, et un rendez-vous de transition avec une infirmière en oncologie pour passer en revue le plan de soins. En mesurant leurs besoins et leur sentiment d'autonomie au départ, puis une année après la transition vers ce projet d'amélioration de la qualité, nous n'avons généralement pas trouvé après l'écoulement de cette année de changements significatifs dans les besoins physiques et psychosociaux ou dans le sentiment d'autonomie chez les survivants du cancer colorectal et les survivantes du cancer du sein. Après un an, les survivants du cancer colorectal présentaient une hausse statistiquement significative de la peur de la récidive. Les infirmières en oncologie sont bien placées pour reconnaitre les besoins uniques des survivants du cancer et elles contribuent à trouver des moyens pour continuer à répondre à leurs besoins en offrant des séances éducatives et des plans de soins de survie individualisés, et en les aidant à se sentir autonomes dans la gestion de leur maladie chronique au moment d'être transférés aux soins primaires. Des recherches ultérieures seront requises pour explorer la contribution des séances d'éducation et des plans de soins de survie individualisés.

\section{RÉFÉRENCES}

Accreditation Canada and Health Standards Organization. Required organizational practices handbook 2017. Ottawa.

Adams, K., Greiner, A.C., \& Corrigan, J. M. (2004). Report of a summit. The 1st annual crossing the quality chasm summit: A focus on communities. Washington, DC: National Academies Press.

Bravo, P., Edwards, A., Barr, P.J., Scholl, I., Elwyn, G., \& McAllister, M. (2015). Conceptualising patient empowerment: A mixed methods study. BMC Health Services Research, 15(1), 252.

Bulsara, C.E., \& Styles, I. (2013). Development of a cancer related patient empowerment scale using the polytomous Rasch measurement model. Cancer and Clinical Oncology, 2(1), 87.

Bulsara, C., Styles, I., Ward, A.M., \& Bulsara, M. (2006). The psychometrics of developing the patient empowerment scale. Journal of Psychosocial Oncology, 24(2), 1-16.

Canadian Cancer Statistics (2016). Canadian Cancer Society, Statistics Canada. Government of Canada. Retrieved from http://www. 
cancer.ca/ /media/cancer.ca/CW/cancer\%20information/ cancer\%20101/Canadian\%20cancer\%20statistics/CanadianCancer-Statistics-2016-EN.pdf?la=en

Cancer Care Ontario Members of the Colorectal Cancer Survivorship Group (2012). Follow-up care, surveillance protocol, and secondary prevention measures for survivors of colorectal cancer. Toronto (ON): Cancer Care Ontario; 2012 Feb 3. Program in Evidence-based Care Evidence-Based Series No.: 26-2 Version 2

Dawes, A.J., Hemmelgarn, M., Nguyen, D.K., Sacks, G.D., Clayton, S.M., Cope, J.R., Maggard-Gibbons, M. (2015). Are primary care providers prepared to care for survivors of breast cancer in the safety net? Cancer, 121(8), 1249-1256.

Drayton, M., Tamblyn, M., Mercer, C., Ontario Agency for Health Protection and Promotion, Public Health Ontario, Cancer Care Ontario. Canadian Electronic Library (Firm). (2012). Taking action to prevent chronic disease: Recommendations for a healthier Ontario. Toronto, ON: Ontario Agency for Health Protection and Promotion.

Eskildsen, N.B., Joergensen, C.R., Thomsen, T.G., Ross, L., Dietz, S.M., Groenvold, M., \& Johnsen, A.T. (2017). Patient empowerment: A systematic review of questionnaires measuring empowerment in cancer patients. Acta oncologica (Stockholm, Sweden), 56(2), 156.

Fitch, M.I. (2008). Supportive care framework. Canadian Oncology Nursing Journal/Revue canadienne de soins infirmiers en oncologie, 18(1), 6-14.doi:10.5737/1181912x181614

Grant, M., De Rossi, S., Sussman, J. (2015). Supporting models to transition breast cancer survivors to primary care: Formative evaluation of a cancer care Ontario initiative. American Society of Clinical Oncology. doi:10.1200/JOP.2015.003822

Hebdon, M., Abrahamson, K., McComb, S., \& Sands, L. (2014). Transitioning patients to survivorship care: A systematic review. Oncology Nursing Forum, 41(6), 615-625.

Howell, D., Hack, T., Oliver, T.K., Chulak, T. Mayo, S., Aubin, M., Chasen, M., ... Tompson, M. (2011). Survivorship services for adult cancer populations: A pan-canadian guideline. Current Oncology, 18(6), 265-281.

Institute of Medicine and National Research Council (2006). From Cancer Patient to Cancer Survivor: Lost in Transition. Washington, D.C: The National Academies Press. Washington D.C.

Jabson, J. (2014). Follow-up care instructions, treatment summaries, and cancer survivors' receipt of follow-up health care and late/long term effects. Support Care Cancer. doi:10.1007/s00520-014-2532-5

Jerofke, T.A. (2013). Concept analysis of empowerment from survivor and nurse perspectives within the context of cancer survivorship. Research and Theory for Nursing Practice: An International Journal, 27(3), 157-172. doi.org:/10.1891/1541-6577.27.3.157

Kane, P., Boland, P., \& Herst, P. (2014). A pathway to empowerment: Evaluating a cancer education and support programme in New Zealand. European Journal of Cancer Care, 23(5), 668-674. doi: 10.1111/ecc.12188

Knobf, T. (2013). Being prepared: Essential to self-care and quality of life for the person with cancer. Clinical Journal of Oncology Nursing, 17(3). doi:10:1188/13.CJON.255-261

Kuijpers, W., Groen, W.G., Aaronson, N.K., \& van Harten, W.H. (2013). A systematic review of web-based interventions for patient empowerment and physical activity in chronic diseases: relevance for cancer survivors. Journal of Medical Internet Research, 15(2).

Lauzier, S., Campbell, H.S., Livingston, P.M., \& Maunsell, E. (2014). Indicators for evaluating cancer organizations' support services: Performance and associations with empowerment. Cancer, 120(20), 3219-3227. doi:10.1002/cncr.28846
Lewis, R.A., Neal, R.D., Williams, N.H., France, B., Hendry, M., Russell, D., \& Wilkinson, C. (2009). Follow-up of cancer in primary care versus secondary care: Systematic review. British Journal of General Practice, 59(564), e234-e247.

Luctkar-Flude, M., Aiken, A., McColl, M.A., \& Tramner, J. (2015). A comprehensive framework and key guideline recommendations for the provision of evidence-based breast cancer survivorship care within the primary care setting. Family Practice, 32(2). 129-140. doi:10.1093/fampra/cmu082

Mayo Clinic Cancer Center (N.D.). Cancer Survivors Survey of Needs. Unpublished instrument, Cancer Education Program of the Mayo Clinic Cancer Center, Rochester, MN.

McAllister, M., Dunn, G., Payne, K., Davies, L., \& Todd, C. (2012). Patient empowerment: The need to consider it as a measurable patient-reported outcome for chronic conditions. BMC Health Services Research, 12(1), 157.

McCarley, P. (2009). Patient empowerment and motivational interviewing: Engaging patients to self-manage their own care. Nephrology Nursing Journal, 36(4), 409-413.

McCorkle, R., Ercolano, E., Lazenby, M., Schulman-Green, D., Schilling, L.S., Lorig, K., \& Wagner, E.H. (2011). Self-management: Enabling and empowering patients living with cancer as a chronic illness. CA: American Cancer Journal for Clinicians, 61, 50-62. doi:10.3322//caac. 20093

Mok, E., Martinson, I., \& Wong, T.K. (2004). Individual empowerment among Chinese cancer patients in Hong Kong. Western Journal of Nursing Research, 26(1), 59-75.

Morgan, M.A. (2009). Cancer survivorship: History, quality-oflife issues, and the evolving multidisciplinary approach to implementation of cancer survivorship care plans. Oncology Nursing Forum, 36(4), 429-436.

Pereira, N. (2014). Educating and empowering colorectal cancer survivors. Gastrointestinal Nursing, 12(9), 33-40.

Phillips, J.L., \& Currow, D.C. (2010). Cancer as a chronic disease. Collegian, 17(2), 47-50.

Polit, D.B., \& Beck, T.C. (2012). Nursing research. Generating and assessing evidence for nursing practice (9th ed.). Philadelphia, PA. Wolters Kluwer. Lippincott Williams \& Wilkins

Rushton, M., Morash, R., Larocque, G., Liska, C., Stocia, L., DeGrasse, C., \& Segal, R. (2015). Wellness beyond cancer program: Building an effective survivorship program. Current Oncology, 22(6), 419434. https://doi.org/10.3747/co.22.2786

Ryhanen, A.M., Rankinen, S. Siekkinen, M. Saarinen, M., Korvenranta, H., \& Leino-Kilpi, H. (2013). The impact of an empowering internet-based breast cancer patient pathway on breast cancer patients' clinical outcomes: A randomized controlled trial. Journal of Clinical Nursing, 22(7-8), 1016-1025.

Schlairet, M., Heddon, M.A., \& Griffis, M. (2010). Piloting a needs assessment to guide development of a survivorship program for a community cancer center. Oncology Nursing Forum, 37(4), 501-508.

van den Berg, S.W., Van Amstel, F.K.P., Ottevanger, P.B., Gielissen, M.F.M., \& Prins, J.B. (2013). The cancer empowerment questionnaire: Psychological empowerment in breast cancer survivors. Journal of Psychosocial Oncology, 31(5), 565-583. doi:10.10 80/07347332.2013.825361

van Uden-Kraan, C.F., Drossaert, C.H., Taal, E., Shaw, B.R., Seydel, E.R., \& van de Laar, M.A. (2008). Empowering processes and outcomes of participation in online support groups for patients with breast cancer, arthritis, or fibromyalgia. Qualitative Health Research, 18(3), 405-417.

Wagner, E.H., Austin, B.T., Davis, C., Hindmarsh, M., Schaefer, J., \& Bonomi, A. (2001). Improving chronic illness care: Translating evidence into action. Health Affairs, 20(6), 64-78. 